\title{
The reliability and validity of the Arabic version of the Mouth Handicap in Systemic Sclerosis (MHISS) Questionnaire in Arabian systemic sclerosis patients
}

\author{
Enas A. Elattar ${ }^{1}$, Mona G. Balata ${ }^{1,2}$ and Reem El Mallah ${ }^{1 *}$ (D)
}

\begin{abstract}
Background: Systemic sclerosis (SSC) is an autoimmune multi-systemic disease with predominant affection of connective tissue. Skin is characteristically affected in SSC and is almost responsible for oral complications as well as aesthetic changes which affect the patient's oral health-related quality of life. The Mouth Handicap in Systemic Sclerosis (MHISS) Questionnaire is the first tool to subjectively calculate the handicap associated with mouth disability in SSC patients. This study aimed to test for reliability, the validity of the Arabic version of the MHISS to be used in Arabian systemic sclerosis.

Results: This study was conducted on 38 females with SSc with a mean age of $48.7 \pm 5.01$ years and a mean disease duration of $6.23 \pm 3.14$ years - 18 patients (47.4\%) have diffuse SSc while 20 (52.6\%) have localised SSc. The mean value of the total MHISS for SSc patients was $12.21 \pm 3.51$, test-retest reliability of the Arabic version was excellent $(I C C=0.991)$ with excellent internal consistency (Chronbach's $a=0.892)$ with significant negative correlation with mouth opening $(r=-0.512, p<0.05)$ and no correlation with the Health Assessment Questionnaire Disability Index (HAQ-DI).

Conclusion: Our results demonstrated that the Arabic version of the MHISS questionnaire had excellent test-retest reliability and very good validity in measuring mouth disabilities in Arabian SSc patients.
\end{abstract}

Keywords: MHISS, Mouth opening, Xerostomia, Systemic sclerosis, Arabic version

\section{Background}

Systemic Sclerosis (SSc) is an autoimmune multisystemic disease with predominant affection of connective tissue with excessive collagen deposition, microvascular damage, and obliteration. It affects mainly the skin joints, tendons as well as internal organs such as the lungs, heart, kidneys, and gastrointestinal tract [1]. Skin is characteristically affected in SSc, where it becomes thick and harder due to infiltration of the dermis by immune cells, mainly $\mathrm{T}$ helper type 2 lymphocytes

\footnotetext{
* Correspondence: reemelmallah@gmail.com

'Rheumatology and Rehabilitation, Faculty of Medicine, Ain Shams University, Cairo, Egypt

Full list of author information is available at the end of the article
}

and macrophages, followed later by fibrosis [2]. Skin involvement in SSc is a keystone for clinical classification of SSc, as patients with fibrosis of the face and limbs (distal to elbows and knees) are classified as limited systemic sclerosis (ISSc), whereas patients with diffuse skin fibrosis involving the trunk and proximal limbs are classified as diffuse systemic sclerosis (dSSc) [3]. The most widely used tool to score skin involvement is the modified Rodnan skin score (mRSS); it is easy, and the reproductive score also reflects the disease activity [4].

Skin involvement of the face is almost responsible for oral complications as well as aesthetic changes such as narrow mouth opening (microstomia), tight shortened frenulum of the tongue, loss of nasolabial fold, thinning 
of lips and nasal alae, and fibrosis of the salivary glands with resultant xerostomia. Fibrosis of the soft tissue around the mandibles and osteolysis of the mandibular angles add more to the microstomia [5]. These orofacial manifestations are typical and characteristic for SSc patients, affecting their oral health-related quality of life (OHRQoL) such as eating, the ability to incise large solid foods, and chewing, as well as speaking especially when associated with xerostomia (sicca syndrome) leading to nutritional affection; moreover, microstomia renders the daily oral hygiene and dental care by the dentist to a challenging mission [6]. Aesthetic changes due to skin fibrosis are considered as one of the most annoying aspects of SSc [7].

Over the years, different scales and questionnaires have been used to assess the global disabilities of SSc patients such as the Health Assessment Questionnaire (HAQ) and the Short form 36 (SF 36) as well as more specific scales to assess hand function and disability using the Hand Mobility in Scleroderma (HAMIS) scale [8] or Duruoz index [9]. They are used to assess the handicaps, evolution, progress of the disease as well as the effect of different medical treatments and interventions. Oral manifestations and OHRQoL are often underestimated or studied, which could be due to the devastating and severe systemic illness of the disease [10].

A specific self-assessment questionnaire has been developed for the assessment of oral disabilities and handicaps in SSc patients which is the Mouth Handicap in Systemic Sclerosis (MHISS) Questionnaire; it is the first tool to subjectively calculate the handicap associated with mouth disability in SSc patients, reflecting also the related oral health quality of life which is not assessed by the usually used HAQ and Short form-36 [11].

The aim of this study was to test for reliability and validity of the Arabic version of the MHISS to be used in Arabian systemic sclerosis patients.

\section{Methods}

\section{Patients}

Adult Arabian literate female patients with an age range of 18-60 years, diagnosed to have SSc according to the American College of Rheumatology (ACR) and European League Against Rheumatism (EULAR) classification criteria [12] for Systemic Sclerosis 2013, and attending the outpatient \& inpatient department of Physical Medicine, Rheumatology and Rehabilitation of Care National Hospital Riyadh, NJCH, Jeddah, Kingdom of Saudi Arabia and Ain Shams University Hospitals, Egypt were asked to participate in this study after giving a written consent. This sample of patients included a variant of Arabian populations (Saudi, Egyptian, Syrian, Sudanese, Lebanese, Yamani, and Tunisian). The study was approved by the local department committee and in accordance with the declaration of Helsinki.

\section{Exclusion criteria}

Patients younger than 18 years, those with other concomitant rheumatological diseases (mixed connective tissue disease, primary sicca syndrome), patients who had any previous aesthetic intervention for their face, pregnant females, and patients with any associated malignancies were excluded from the study.

The sample size was initially calculated on the collected 60 cases, with a confidence level of $90 \%$ and a $10 \%$ margin of error; the ideal sample was 32 cases (http://www.quattrics.com).

\section{Clinical assessment}

All the patients who accepted to participate in the study and gave a written consent (42 females and 2 males) underwent full medical history and examination with great focus on the disease duration, skin involvement, and classification to either subtypes according to international classifications [12]; Raynaud's phenomenon; digital ulcers; hypo and hyperpigmentations; telangiectasia; and musculoskeletal involvement such as arthritis or arthralgia and flexion contractures.

Skin thickness was assessed with modified Rodnan skin score (mRss) [4], mouth opening was measured as the maximum distance between the tips of right upper and lower incisors in centimetres (a mean of two measures was used). A series of investigations were done (or recorded from the patients' electronic or hard files) for exploring the internal organ affection such as plain chest $\mathrm{X}$-ray and high-resolution computer tomography; pulmonary function tests to detect interstitial lung disease; and colour Doppler echocardiography to measure pulmonary hypertension and other cardiac involvements the could be present as pericarditis \& left ventricular failure. Oesophageal hypomotility was tested with barium radiography; xerostomia was subjectively recorded on a visual analogue scale (VAS) from $0-10$, where 0 indicated no symptoms of dry mouth and 10 was the maximum sense of mouth dryness [13].

\section{Translation procedure}

Translation of the MHISS Questionnaire was performed according to the Beaton guidelines [14]. Two translations from English to classic Arabic were done by two independent native Arabic speakers who are fluent in English as well; both had knowledge about the purpose of the study for better adaptation and equivalence between the original version and translated version of the questionnaire with a pooling in one translation form, which is then retranslated back to English version by an independent bilingual translator who was completely 
blind to the original English version of the questionnaire. Comparison was done to the original MHISS to check for similar consistency and to ensure that the Arabic version is equivalent to the original English version, and to test for comprehensibility, the Arabic questionnaire was tested on 20 healthy individuals (not health caregivers), ten patients with SSc disease, and another 5 patients with any other rheumatic illness than SSc. All cases reported that they understood the questions easily, then a final formulation of the Arabic version of the questionnaire was created to be used in the study.

\section{Questionnaire administration}

All the patients who accepted to participate in the study were administered the Arabic version of Mouth Handicap in Systemic Sclerosis (MHISS), which consists of 12 items; each item is rated from 0 to 4 , with a total score of 48 and divided into three subscales: subscale 1 (5 items; $1,3,4,5$, and 6) which measures handicap related to reduced mouth opening, subscale 2 (5 items; 2, 7, 8, 9 , and 10) which measures handicap related to sicca syndrome, and subscale 3 (2 items; 11 and 12 ) which assesses the aesthetic affection [11]; patients were asked to answer the questionnaire again after 2 weeks to assess the test-retest reliability. Only 38 females came after 2 weeks, and the other 4 females and 2 males could not be reached.

The patients were administered the Arabic version of the Health Assessment Questionnaire disability index (HAQ-DI) which has been validated previously [15]; it consists of 20 categories to be self-answered by the patients. They are organised in 8 items: dressing and grooming, arising, eating, walking, personal hygiene, reaching, gripping, and other activities. Each category is rated from 0 (no difficulty) to 3 (unable to do). A score for each category is the highest score for any question in the category. A disability index is calculated by adding the scores from each category and dividing by the number of categories answered and rated from 0 (less disabled) to 3 (more disabled) [16]. As regards the treatment, it was tailored for each patient; in most cases, it was a combination of steroids, azathioprine, methotrexate, ACE inhibitors, nifedipine, and sildenafil. It was difficult to categorize the patients into groups according to the treatment to study the effect of these combination on the score of the questionnaire. In addition, all previous studies for validation of this questionnaire did not include the medications in their testing parameter for reliability or external validity.

\section{Statistics}

All data were collected, tabulated, and statistically analysed. Analysis of data was done by SPSS (Statistical Program for Social Sciences) as follows:
-Description of quantitative variables as mean, SD, and range as the test of normality (Alamogordo-Smirnov) showed normal data distribution. Description of qualitative variables as number and percentage;

-Student's $t$ test was used to compare groups as regards quantitative variables; the $p$ value was considered significant if $\leq 0.05$;

-Chi-square test was used to compare groups as regards qualitative variables; test-retest reliability was analysed by using Spearman's $\rho$ correlation coefficient (ICC). The internal consistency of the Arabic version of MHISS was assessed by using Cronbach's $\alpha$ coefficient.

-The validity of the Arabic version of MHISS was studied and assessed by correlating its total scores with disease duration, maximal mouth opening, Rodnan skin score, HAQ-DI, ILD, PAH, kidney affection, and xerostomia measured on VAS by using Spearman's $\rho$ correlation coefficients. Spearman's $\rho$ correlation coefficients ranging between 0.81 and 1.00 were considered excellent, while ranges between 0.61 and $0.80,0.41$ and 0.60 , 0.21 and 0.40 , and 0 and 0.20 were accepted as very good, good, weak, and bad, respectively [17].

\section{Results}

This study was conducted on 38 females; their descriptive data are shown in Table 1.

The mean of the total MHISS scores of the patients was $12.21 \pm 3.51$, and its mean values in different subscales were $6.15 \pm 1.60,4.6 \pm 1.90$ and $1.36 \pm 1.19$ for subscales 1,2 , and 3 , respectively.

The mean value of total MHISS was not significantly different between dSSc and ISSc and those with kidney affection, but was significantly higher in patients with positive ILD, PAH, positive oesophageal affection, positive musculoskeletal affection, and positive digital ulcer as shown in Table 2.

Test-retest reliability of the Arabic version of MHISS (three subscales) was studied with Spearman's $p$ correlation coefficients, where ICC of the total score was 0.991 and for the subscales were 0.993, 0.98, and 0.991 for subscales 1,2 , and 3 , respectively, which are considered excellent correlation, as shown in Table 3.

Internal consistency of the total Arabic version of MHISS which consists of 12 items (three subscales) was tested with Chronbach's $\alpha$ values, which showed an excellent internal consistency for the total (0.892) and subscales 1 (0.854), 2 (0.905), and 3 (0.888), respectively.

On studying the external validity of the Arabic version of MHISS, there was a significant correlation between the total HMISS and disease duration $(r=0.528, p<0.001)$, significant negative correlation with mouth opening $(r=-0.512, p<0.05)$ as well as significant correlation with xerostomia measured on VAS $(r$ 0.577, $p<$ $0.001)$. However, there was no correlation with age of 
Table 1 Descriptive data of patients

\begin{tabular}{|c|c|c|}
\hline & & Values \\
\hline \multirow[t]{2}{*}{ Age } & Range & $(40-60)$ \\
\hline & Mean \pm SD & $48.71 \pm 5.01$ \\
\hline \multirow[t]{2}{*}{ Disease duration } & Range & $(1-13)$ \\
\hline & Mean \pm SD & $6.23 \pm 3.14$ \\
\hline \multicolumn{3}{|l|}{ Subtype: } \\
\hline dSSC & No. (\%) & $18(47.4 \%)$ \\
\hline ISSC & №. (\%) & $20(52.6 \%)$ \\
\hline \multirow[t]{2}{*}{ Mouth opening $(\mathrm{cm})$} & Range & $(2-4)$ \\
\hline & Mean \pm SD & $3.07 \pm 0.50$ \\
\hline \multirow[t]{2}{*}{ Skin score (MRSS) } & Range & $(9-25)$ \\
\hline & Mean \pm SD & $15.23 \pm 4.21$ \\
\hline \multirow[t]{2}{*}{ HAQ-DI } & Range & $(0.4-1.7)$ \\
\hline & Mean \pm SD & $0.97 \pm 0.36$ \\
\hline \multirow[t]{2}{*}{ Xerostomia (VAS) } & Range & $(1-9)$ \\
\hline & Mean \pm SD & $3.39 \pm 2.09$ \\
\hline \multirow[t]{2}{*}{ Total MHISS } & Range & $(7-19)$ \\
\hline & Mean \pm SD & $12.21 \pm 3.51$ \\
\hline \multirow[t]{2}{*}{ Subscale 1} & Range & $(4-10)$ \\
\hline & Mean \pm SD & $6.15 \pm 1.60$ \\
\hline \multirow[t]{2}{*}{ Subscale 2} & Range & $(2-10)$ \\
\hline & Mean \pm SD & $4.6 \pm 1.90$ \\
\hline \multirow[t]{2}{*}{ Subscale 3} & Range & $(0-4)$ \\
\hline & Mean \pm SD & $1.36 \pm 1.195$ \\
\hline ILD & No. (\%) & $15(39.5 \%)$ \\
\hline PAH & No. (\%) & $9(23.7 \%)$ \\
\hline Kidney affection & No. (\%) & $2 / 38(0.05 \%)$ \\
\hline Oesophageal affection & No. (\%) & $17(44.7 \%)$ \\
\hline Musculoskeletal affection & No. (\%) & $15(39.5 \%)$ \\
\hline Raynaud's phenomenon & No. (\%) & $38(100 \%)$ \\
\hline Telangiectasia & No. (\%) & $10(26.3 \%)$ \\
\hline Digital ulcer & No. (\%) & $11(28.9 \%)$ \\
\hline Hypo and hyper pigmentation & No. (\%) & $5(13.2 \%)$ \\
\hline
\end{tabular}

$H A Q$ Health assessment questionnaire, VAS visual analogue scale, $d S S c$ diffuse systemic sclerosis, ISSC localized systemic sclerosis, ILD interstitial lung disease, $P A H$ pulmonary artery hypertension, MHISS Mouth Handicap in

Systemic Sclerosis

the patients, skin score (MRSS), kidney affection, ILD, $\mathrm{PAH}$, kidney affection, or HAQ-DI as shown in Table 4.

\section{Discussion}

The aim of this study was to test for validity and reliability of the Arabic version of the Mouth Handicap in Systemic Sclerosis (MHISS) Questionnaire, to be used for assessing the Oral Health-Related Quality of Life (OHRQoL) in Arabian Systemic Sclerosis
Table 2 The mean value of total MHISS in the major clinical manifestations of the disease

\begin{tabular}{|c|c|c|c|c|}
\hline & \multirow[t]{2}{*}{ No. } & \multirow{2}{*}{$\begin{array}{l}\text { Total MHISS } \\
\text { Mean } \pm \text { SD }\end{array}$} & \multicolumn{2}{|l|}{$t$ test } \\
\hline & & & $t$ & $p$ value \\
\hline \multicolumn{5}{|l|}{ Subtype } \\
\hline dSSC & 18 & $12.2 \pm 3.38$ & 0.019 & 0.980 \\
\hline ISSC & 20 & $12.1 \pm 3.72$ & & \\
\hline \multicolumn{5}{|l|}{ ILD } \\
\hline Negative & 23 & $11.17 \pm 3.01$ & -2.387 & $0.022^{*}$ \\
\hline Positive & 15 & $13.8 \pm 3.74$ & & \\
\hline \multicolumn{5}{|l|}{ PAH } \\
\hline Negative & 29 & $11.37 \pm 3.15$ & -2.855 & $0.007^{*}$ \\
\hline Positive & 9 & $14.88 \pm 3.44$ & & \\
\hline \multicolumn{5}{|c|}{ Kidney affection } \\
\hline Positive & 2 & $12.9 \pm 3.36$ & 0.018 & 0.990 \\
\hline Negative & 37 & $12.8 \pm 3.92$ & & \\
\hline \multicolumn{5}{|c|}{ Oesophageal affection } \\
\hline Negative & 21 & $10.90 \pm 2.68$ & -2.760 & $0.009^{*}$ \\
\hline Positive & 17 & $13.82 \pm 3.82$ & & \\
\hline \multicolumn{5}{|c|}{ Musculoskeletal affection } \\
\hline Negative & 23 & $10.52 \pm 2.50$ & -4.525 & $0.000^{*}$ \\
\hline Positive & 15 & $14.8 \pm 3.32$ & & \\
\hline \multicolumn{5}{|l|}{ Telangiectasia } \\
\hline Negative & 28 & $11.57 \pm 3.30$ & -1.942 & 0.060 \\
\hline Positive & 10 & $14 \pm 3.65$ & & \\
\hline \multicolumn{5}{|l|}{ Digital ulcer } \\
\hline Negative & 27 & $11.25 \pm 3.13$ & -2.851 & $0.007^{*}$ \\
\hline Positive & 11 & $14.54 \pm 3.44$ & & \\
\hline \multicolumn{5}{|c|}{ Hypo and hyperpigmentation } \\
\hline Negative & 33 & $12.33 \pm 3.62$ & 0.547 & 0.588 \\
\hline Positive & 5 & $11.4 \pm 2.88$ & & \\
\hline
\end{tabular}

dSSC diffuse systemic sclerosis, ISSC localised systemic sclerosis, ILD interstitial lung disease, $P A H$ pulmonary artery hypertension, MHISS Mouth Handicap in Systemic Sclerosis, $t \mathrm{t}$ value of Student's $t$ test,

${ }^{*} p$ value; significant if $\leq 0.05$

Table 3 Test-retest reliability of the Arabic version of MHISS

\begin{tabular}{lllllll}
\hline Reliability & & Test & Retest & \multicolumn{2}{c}{ Correlation } \\
\cline { 3 - 6 } & & & & $r$ & $p$ value \\
\hline Total MHISS & Range & $7-19$ & $8-19$ & 0.993 & $<0.001^{*}$ \\
& Mean \pm SD & $12.21 \pm 3.519$ & $12.184 \pm 3.399$ & & \\
Subscale 1 & Range & $4-10$ & $4-10$ & $0.985<0.001^{*}$ \\
& Mean \pm SD & $6.157 \pm 1.603$ & $6.237 \pm 1.601$ & & \\
Subscale 2 & Range & $2-10$ & $2-9$ & $0.991<0.001^{*}$ \\
& Mean \pm SD & $4.68 \pm 1.905$ & $4.605 \pm 1.793$ & & \\
Subscale 3 & Range & $0-4$ & $0-4$ & $0.991<0.001^{*}$ \\
& Mean \pm SD & $1.368 \pm 1.195$ & $1.342 \pm 1.214$ & & \\
\hline
\end{tabular}

MHISS Mouth Handicap in Systemic Sclerosis, $r$ Spearman's correlation (ICC) ${ }^{*} p$ value; significant if $\leq 0.05$ 
Table 4 External validity of the Arabic version of MHISS

\begin{tabular}{lll}
\hline Correlation & \multicolumn{2}{l}{ Total MHISS } \\
\cline { 2 - 3 } & $r$ & $p$ value \\
\hline Age & 0.167 & 0.316 \\
Duration & 0.528 & $0.001^{*}$ \\
Mouth opening & -0.512 & $0.05^{*}$ \\
Skin score (MRSS) & 0.050 & 0.767 \\
ILD & 0.158 & 0.315 \\
PAH & 0.112 & 0.491 \\
Kidney affection & 0.125 & 0.467 \\
HAQ-DI \% & 0.113 & 0.500 \\
Xerostomia (VAS) & 0.577 & $0.000^{*}$
\end{tabular}

MHISS Mouth Handicap in Systemic Sclerosis, MRSS modified Rodnan skin score, ILD interstitial lung disease, $P A H$ pulmonary artery hypertension, HAQ-DI $\%$ Health Assessment Questionnaire disability index, $r$ Spearman's correlation $V A S$ visual analogue scale

${ }^{*} p$ value significant if $\leq 0.05$

patients, as these oral affections are not measured by the usual HAQ and SF-36 Questionnaire.

The study was conducted on 38 adult Arabian literate females with a mean age of $48.71 \pm 5.01$ years and mean disease duration of $6.23 \pm 3.14$ years. Eighteen patients (47.4\%) have diffuse SSc, while 20 patients $(52.6 \%)$ have localised SSc. The mean of the total MHISS score was $12.21 \pm 3.51$, which is less than that of other studies, where the scores for the original French, Italian, Dutch, and Turkish versions of MHISS were 18.8 \pm 10.2 , $17.65 \pm 5.20,20.3 \pm 9.7$, and $22.4 \pm 10.72$, respectively [11, 18-20]. This could be due to ethnic variance especially that the tested patients are of different ethnic groups, yet all are Arabian, or due to climate difference; however, there are no studies that showed the climate influence on the MHISS score. The total score was not significantly different between dSSc and ISSc (mean 12.2 versus 2.1, $p=0.98$ ) which is in accordance with the Dutch version where they found no significant difference between $\mathrm{dSSc}$ and $1 \mathrm{SSc}$ (mean 17.2 versus 17.8, $p=$ 0.842 ). However, this result is different from the Italian study, where they stated that the total score and the score of subscale 2 (handicap related to sicca syndrome) were higher in $\mathrm{dSSc}$ than in ISSc patients, and they explained that result by the higher prevalence of sicca syndrome in dSSc in respect to $1 S S c$ [18].

Test-retest reliability analysis of the Arabic version gave ICC of 0.99 which is almost similar to the original French (0.96) [11], Italian (0.93) [18], Dutch (0.94) [19], and Turkish (0.88) [20] versions of MHISS, which indicates that the tested items within the questionnaire are stable.

On testing the internal consistency of the Arabic version of MHISS questionnaire with Cronbach's $\alpha$ values, they were 0.892 for the total score and $0.854,0.905$, and
0.888 for subscales 1,2 , and 3 , respectively, which is in great consistency with the Dutch version, where their values were 0.88 for the total score and 0.86 and 0.79 for subscales 1 and 2, respectively [19]. Our values were near to the values of the Italian version [18], as they reported that Cronbach's $\alpha$ value was 0.99 for the total score, while the Turkish version reported the Cronbach's $\alpha$ values were 0.86 for the total score and $0.92,0.905$. and 0.78 for the subscales 1,2 , and 3, respectively [20]. These data recorded from different versions indicate that the items are consistent even with translation. We studied the external validity of the Arabic version of MHISS, and there was a significant negative correlation with mouth opening $(r=-0.512, p<0.05)$ indicating the value of MHISS in assessing the decreased mouth opening and the related oral health complications in SSc patients. This correlation was similar to the results of the original French [11], Italian [18], Dutch [19], and Turkish [20] versions of MHISS, where they reported a significant correlation of the total MHISS and the mouth opening $(r=0.34,-0.38,-0.51,-0.73$, respectively). Moreover, in our study of the validity of the MHISS and the oral xerostomia measured on the VAS scale $(0-10)$, there was a significant positive correlation $(r 0.577, p<0.001)$, which was again similar to the results of the Dutch version of MHISS, where they found a positive correlation with subjective xerostomia $(r=0.53, p<0.001)$ [19]. However, there was no correlation with HAQ-DI $(r=$ $0.11, p>0.05)$ which is following the previous Italian validation study, where they found no correlation between MHISS and HAQ [18], in contrast to the original French [11], Dutch [19], and Turkish [20] versions which showed a positive correlation with HAQ. The lack of correlation between MHISS and HAQ-DI in our study is accepted as the MHISS measures of health aspects not assessed with HAQ, such as reduced mouth opening, dryness of the mouth, as well as aesthetic features which are the main OHRQol in SSc patients. Moreover Baron et al. showed that the Oral HRQoL in SSc is independently associated with global HRQoL, and it is not detected in the assessment of disease severity in SSc, although they did not use the MHISS score; but they suggested that OHRQol is an additional dimension of HRQoL which is not detected by global HRQol as SF-36 [21]. No significant correlation was found with the total MHISS score and different disease aspects such as ILD, PAH, and kidney affection which was reported previously [11]. These results highlight the need for the MHISS as a specific questionnaire concerned with mouth opening difficulties, dryness of mouth, and aesthetic facial concern to be validated and used in routine care and follow up of SSc patients, as it was used previously to study the effect of some rehabilitation techniques such as the Kabat technique, facial physiotherapy, 
and home exercise for SSc patients. These studies documented improvement of the mouth opening recorded on the MHISS score while the HAQ assessment did not show any change $[22,23]$.

\section{Limitations}

Only females were tested, and there was the inability to study each ethnic group separately due to the small sample size.

\section{Conclusion}

The Arabic version of MHISS is a self-answered questionnaire, saving physicians' time in translating the questionnaire to the patients. It is reliable and valid for assessing mouth handicap in variant populations of Arabian SSc patients. The value of the questionnaire as a tool in the long-term follow up should be tested in future studies.

\section{Supplementary information}

Supplementary information accompanies this paper at https://doi.org/10. 1186/s43166-020-00038-x.

Additional file 1.

\section{Abbreviations}

SSc: Systemic sclerosis; dSSc: Diffuse systemic sclerosis; mRss: Modified Rodnan skin score; OHRQoL: Oral health-related quality of life; HAQ: Health Assessment Questionnaire; SF 36: Short form 36; HAMIS: Hand mobility in scleroderma; MHISS: Mouth Handicap in Systemic Sclerosis

\section{Acknowledgements}

Thanks to Mr. Abdelaziz Mustafa El-Garf (aziz_stat@yahoo.com) for his great effort in preparing the statistical analysis of this work.

\section{Authors' contributions}

EE gave idea, put study design, and statistically analyse it. All authors (EE, BM, and ER) collected the patients' data. EE and ER wrote the paper and revised it. ER prepared it for publication. The authors read and approved the final version of the manuscript.

\section{Funding}

This study had no funding from any resource.

\section{Availability of data and materials}

The datasets used and/or analysed during the current study are available from the corresponding author on reasonable request.

\section{Ethics approval and consent to participate}

This study was approved by the Ethical Committee of Scientific Research the Research Ethics Committee of Care National Hospital in Riyadh, Kingdom of Saudi Arabia (date formed: 01/01/2019; reference number of approval: $\mathrm{CNH}-$ 021).

All patients included in this study gave written informed consent to participate in this research. If the patient was less than 16 years old or unconscious at the time of the study, written informed consent for their participation was given by their parent or legal guardian.

\section{Consent for publication}

The content of the manuscript has not been published, or submitted for publication elsewhere. All patients included in this research gave written informed consent to publish the data contained within this study. If the patient was less than 16 years old, deceased, or unconscious when consent for publication was requested, written informed consent for the publication of this data was given by their parent or legal guardian.

\section{Competing interests}

The authors declare that they have no competing interests.

\section{Author details}

${ }^{1}$ Rheumatology and Rehabilitation, Faculty of Medicine, Ain Shams University, Cairo, Egypt. ${ }^{2}$ Rehabilitation in Care National Hospital, Riyadh, Kingdom of Saudi Arabia.

Received: 17 June 2020 Accepted: 18 August 2020

Published online: 11 September 2020

References

1. Servettaz A, Agard C, Tamby M, Guilpain P, Guillevin P, Guillevin L, Mouthon L (2006) Systemic sclerosis: pathophysiology of a multifaceted disease. Presse Med 35(12):1903-1915. https://doi.org/10.1016/s0755-4982(06)74924-

2. Hassan WA, Hamaad GA, Sayed EA, El Behisy MM, Gomaa MK (2019) Clinical significance of interleukin 27 serum concentration in patients with systemic sclerosis: relation to clinical, laboratory and radiological parameters. Egypt Rheumatol Rehabil 46:101-107 http://www.err.eg.net/text.asp?2019/46/2/1 01/256502

3. Barsotti S, Bellando Randone S, Guiducci S, DellaRossa A (2014) Systemic sclerosis: a critical digest of the recent literature. Clin Exp Rheumatol 32: S194-S205. PMID: 25372802.

4. Clements PJ, Hurwitz EL, Wong WK, Seibold JR, MayesM WB et al (2000) Skin thickness score as a predictor and correlate of outcome in systemic sclerosis. Arthritis Rheum 43:2445-2454. https://doi.org/10.1002/15290131(200011)43:11<2445::AID-ANR11>3.0.CO;2-Q

5. Ferreli C, Gasparini G, Parodi A, Cozzani E, Rongioletti F, Atzori L et al (2017) Cutaneous manifestations of scleroderma and scleroderma-like disorders: a comprehensive review. Clin Rev Allergy Immunol 2017; 53: 306-336. DOI: https://doi.org/10.1007/s12016-017-8625-4

6. Albilia JB, Lam DK, Blanas N, Clokie C, Sandor G et al (2007) Smallmouths... big problems? A review of scleroderma and its oral health implications. J Can Dent Assoc 73:831_836. PMID: 18028759.

7. Amin K, Clarke A, Sivakumar B, Puri A, Fox Z, Brough V, Denton C, Peter E, Butler $P$ et al (2011) The psychological impact of facial changes in scleroderma. Psychol Health Med 16:304-312. https://doi.org/10.1080/ 13548506.2010.540250

8. Sandqvist G, Eklund M (2000) Validity of HAMIS: a test of hand mobility in scleroderma. Arthritis Care Res 13:382-387. PMID: 14635314.

9. Brower LM, Poole $J \mathrm{~L}$ (2004) Reliability and validity of the Duruoz hand index in persons with systemic sclerosis (scleroderma). Arthritis Rheum 51:805809. https://doi.org/10.1002/art.20701

10. Del Rossol A, Bongi S (2014) Oral health in patients with systemic sclerosis. Rheumatology 53:1355-1356. https://doi.org/10.1093/rheumatology/keu179

11. Mouthon L, Rannou F, Bérezné A, Pagnoux C, Arene J-P, Fois E, Cabane J et al (2007) Development and validation of a scale for mouth handicap in systemic sclerosis: the Mouth Handicap in Systemic Sclerosis scale. Ann Rheum Dis. 66(12):1651-1655. https://doi.org/10.1136/ard.2007.070532

12. Van den Hoogen F, Khanna D, Fransen J et al (2013) Classification criteria for systemic sclerosis: an American College of Rheumatology/ European League against Rheumatism collaborative initiative. Ann Rheum Dis 72: 1747-1755. https://doi.org/10.1136/annrheumdis-2013-204424

13. Vitali C, Bombardieri S, Jonsson R, Moutsopoulos H, Alexander E, Carsons S et al (2002) Classification criteria for Sjögren's syndrome: a revised version of the European criteria proposed by the American-European Consensus Group. Ann Rheum Dis 61:554-558. https://doi.org/10.1136/ard.61.6.554

14. Beaton DE, Bombardier C, Guillemin F, Ferraz M (2000) Guidelines for the process of cross-cultural adaptation of self-report measures. Spine 25(24): 3186-3191. https://doi.org/10.1097/00007632-200012150-00014

15. El Meidany Y, EIGaafary M, Ahmed I (2003) Cross-cultural adaptation and validation of an Arabic Health Assessment Questionnaire for use in rheumatoid arthritis patients. Joint Bone Spine 70(3):195-202. https://doi. org/10.1016/s1297-319x(03)00004-6

16. Poole JL, Steen VD (1991) The use of the health assessment questionnaire (HAQ) to determine physical disability in systemic sclerosis. Arthritis Care Res 4:27-31. https://doi.org/10.1002/art.1790040106 
17. Feise RJ, Menke JM (2001) Functional rating index: a new valid and reliable instrument to measure the magnitude of clinical change in spinal conditions. Spine 26(1):78-87. https://doi.org/10.1097/00007632-20010101000015

18. Bongi SM, Del Rosso A, Miniati I et al (2012) The Italian version of the Mouth Handicap in Systemic Sclerosis scale (MHISS) is valid, reliable and useful in assessing oral health-related quality of life (OHRQoL) in systemic sclerosis (SSc) patients. Rheumatol Int 32(9):2785-2790. https://doi.org/10. 1007/s00296-011-2049-x

19. Schouffoer AA, Strijbos E, Schuerwegh AJM et al (2013) Translation, crosscultural adaptation, and validation of the Mouth Handicap in Systemic Sclerosis questionnaire (MHISS) into the Dutch Language. Clin Rheumatol 32(11):1649-1655. https://doi.org/10.1007/s10067-013-2321-4

20. Tore NG, Sari F, Tuna Z, Kucuk H et al (2020) Translation, validation and cross-cultural adaptation of the mouth handicap in systemic sclerosis questionnaire into the Turkish language. International Journal of Rheumatic Diseases. https://doi.org/10.1111/1756-185X.13812

21. Baron M, Hudson M, Tatibouet S, Steele R, Lo E, Gravell S et al (2015) The Canadian Systemic Sclerosis Oral Health Study II: the relationship between oral and global health-related quality of life in systemic sclerosis. Rheumatology 54:692-696. https://doi.org/10.1093/rheumatology/keu396

22. Bongi M, Landi G, Del Rosso A et al (2011) The rehabilitation of facial involvement in systemic sclerosis: efficacy of the combination of connective tissue massage, Kabat's technique and kinesitherapy: a randomized controlled trial. Rheumatol Int 31(7):895-901. https://doi.org/10.1007/s00296010-1382-9

23. Elattar E, Younes T, Hosny S, Hassan M (2014) Value of combined facial rehabilitation techniques and temporomandibular osteopathy in a cohort of Egyptian patients with systemic sclerosis. Life Science Journal 11:12. https:// doi.org/10.7537/marslsj111214.112

\section{Publisher's Note}

Springer Nature remains neutral with regard to jurisdictional claims in published maps and institutional affiliations.

\section{Submit your manuscript to a SpringerOpen ${ }^{\circ}$ journal and benefit from:}

- Convenient online submission

- Rigorous peer review

- Open access: articles freely available online

- High visibility within the field

- Retaining the copyright to your article

Submit your next manuscript at $\boldsymbol{\nabla}$ springeropen.com 